\title{
Application of Parallel Computing to Obtain all Real Solutions of a High Degree Univariate Polynomial Equation
}

\author{
Liying Wang \\ School of Information Science and Technology, Bohai University, Jinzhou, 121013, China \\ 986558686@qq.com
}

Keywords: parallel computing; global convergence; a high degree univariate polynomial equation

\begin{abstract}
The efficient method, which combines the advantages of parallel computing and golden section, is put forward to solve a high degree univariate polynomial equation. This method can be used to overcome the shortcomings of common methods, which need to good initial values and may omit part of real solutions. Firstly, a simulation algorithm are provided. The golden section method is used to reduce the number of iterations and the parallel computing can efficiency calculate the solutions. Then, the stability and convergence of the method are strictly proved. Finally, numerical computations are employed to verify the proposed method. The results indicate that the proposed method can effectively improve the efficiency of solutions and obtain all the real solutions of the equation. The approach has high convergence rate and precision. It can be applied to the large scale problems arising from scientific and engineering computing.
\end{abstract}

\section{Introduction}

A high degree univariate polynomial equation is one form of nonlinear equations, which is so prevalent that it deserves special attention. It is often applied to natural life and practical engineering fields, including power system calculation, automation and so on. For example, using partial differential equation for solving partial differential equations is transformed into high degree univariate polynomial equations. Commonly used to solve such problems is Newton method[1,2,3]and improved algorithm based on Newton method, including the Chebyshev-Halley iteration[4], Super-Hally iteration[5], Jarratt iteration[6]and so on. The methods have the characteristics of quadratic convergence, but such methods generally exist problems are as follows: First, it depends on the initial value. For complex high order equation, it is very difficult to find it, even without any proper initial solution. Second, for such a local convergence of the method, if the initial value is not appropriate and will not be reliable convergence. Third, in the actual application fields, they cannot get all the real solutions to its application is limited.

Aiming at the shortcomings of Newton method and its improved methods, parallel computing is put forward to solving the high order equations. At the same time, to effectively reduce the number of iterations, the golden section method is adopted to iterate. This paper presents five groups of numerical experiments. They show that the proposed method has better performance of obtaining all solutions and computing speed. This method has ability to deal with error automatically which can guarantee solutions not lost in theory and practical computing and get high accuracy. In this paper, stability and convergence of this method is proved by the strict.

\section{The Approach}

In this paper, for the derivation of equation from as follows:

$$
F(x)=\sum_{j=0}^{k} a_{j} x^{j}=0
$$

Where $F(x): x \in D \subset R^{n} \rightarrow R^{n}$ are continuously differentiable mappings in $D, x \in D, F(x)=0$. As a first derivation form $f(x)=0$, the iterative formula for derivative is 


$$
\frac{d^{j} f(x)}{d x^{j}}=\sum_{j=0}^{n} j a_{j} x^{j-1}=0
$$

derivation for $k-1$ order equation is the stagnation points of $\mathrm{k}$ order equation, which can determine the range of the solution of the real number. Then search the real number solution in each interval.

The golden section[7] method is widely used in optimization calculation with the fast convergence speed. Its basic idea is: for $F(x)=0$, which is continuous function and $x \in[a, b]$ is the only one real root. Given $x_{1}=a, x_{2}=b$, if $F\left(x_{1}\right)=0$ or $F\left(x_{2}\right)=0$, then $x=x_{1}$ or $x=x_{2}$. Else comparative $F\left(x_{1}\right), F\left(x_{2}\right)$, if $F\left(x_{1}\right)>F\left(x_{2}\right), x_{1}=b-0.618(b-a)$. On the contrary case, $F\left(x_{1}\right)<F\left(x_{2}\right), x_{2}=a+0.618(b-a)$. So the original interval is short. In new interval repeat the above process, directly ask a root range within the scope of the precision, can get the real root.

\section{Parallel Computing}

Parallel computing can meet the needs of high precision science[8] and engineering computation, and ensure the high efficiency of mass data processing[9]. Huge computational tasks can be divided into sub tasks at the same time[10], these sub tasks are independent of each other, but each other have connection again, many child tasks in parallel execution, so as to complete the calculation of the problem. Nowadays, parallel computing is widely used in electronic commerce, weather forecast and the analysis of the astronomical data, etc. It in the largest extent, guarantee the accuracy of the real-time processing of data at the same time[11], effectively speed up the calculation, the optimization process and fast to solve complex large-scale computing problems.

The traditional serial technology requires more time and low efficiency. Parallel computing combined with the golden section can significantly improve the computational efficiency. For parallel computing, the establishment of $N$ threads. Assuming that iteration data $M$ to store in two-dimensional array, then $M=k N$. The algorithm is carried out task partitioning, repeated child tasks in parallel manner. The thread will be assigned to the next interval to search solution after completing calculation. The algorithm is as follows:

Step one: defined the precision of solutions $\varepsilon=11$, initialize array $a_{1}[M][M], a_{2}[M][M]$;

Step two: The coefficients of the differential equation are stored in $a_{1}[M][M]$, to obtain the derivative equation form is $a x^{2}+b=0$, and find out the solutions to save to $a_{2}[M][M]$;

Step three: To $\left(-\infty, x_{1}\right),\left(x_{1}, x_{2}\right), \cdots,\left(x_{j},+\infty\right)$, using the golden section method, multiple processes executing at the same time to search the solutions.

\section{Stability}

Lemma 1[12] Function $F(x)$ in the closed interval continuous on $[a, b], F(a) \cdot F(b)<0$, then in the open interval $(a, b)$, there are at least one number such as $\zeta, F(\zeta)=0$.

Lemma 2[12] If the function $F(x)$ in the closed interval continuous on $[a, b]$, in the open interval $(a, b)$ can guide, then within $(a, b)$ there are at least a number $\mathrm{M}$, the equation was established.

Proposition If $[a, b]$ has the solutions of $x$, then $x$ is the only real number solution in the interval.

Proof. According to the formula(2) to get the stagnation point $a, b$ of formula(1)according to lemma 1 , if $F(a), F(b)$ has different number, there is a solution in $[a, b]$. Then prove that the function $F(x)$ in the interval $[a, b]$ has the monotonicity. Function $F(x)$ in continuous on $[a, b]$ take $x_{1}, x_{2}$, according to lemma 2 ,

$$
F\left(x_{2}\right)-F\left(x_{1}\right)=f(\zeta)\left(x_{2}-x_{1}\right)\left(x_{1}<\zeta<x_{2}\right)
$$

$x_{2}-x_{1}>0$, if derivative $f(x)$ keep a plus, the $f(\zeta)<0$, then formula (3) the symbol for plus, $F\left(x_{1}\right)<F\left(x_{2}\right)$ which means that the function $F(x)$ on $[a, b]$ monotone increasing. On the 
closed interval $[a, b]$, the function $F(x)$ has monotonicity,. Set $\zeta \in[a, b]$ is a root of $F(x), F(\zeta)=0$, according to the strict monotonicity of function condition, $\forall x \in[a, b], x \neq \zeta$, then $F(x) \neq F(\zeta)=0$, so the only real root in the existence. By the same token when the function is monotone decreasing function only one real root.

\section{The Convergence}

Theorem Set function $y=F(x)$ domain for $\mathrm{D}$, D is the area of two adjacent poles $\mathrm{x} 1$, $\mathrm{x} 2$ (the two extreme value point corresponding to the $y$ value of symbols opposite), the area shall be carried out in accordance with the golden section method iteration, set the n-th iterative get points for $x_{n}$, then $\lim _{n \rightarrow \infty} y=F\left(x_{n}\right)=0$.

Proof. The domain is defined as $D=\left[x_{1}, x_{2}\right], y_{1}=F\left(x_{1}\right), y_{2}=F\left(x_{2}\right)\left(y_{1}, y_{2}\right.$ has the opposite sign), interval length is $l=x_{2}-x_{1}$, division for the first time, after comparing $y_{1}, y_{2}$ the size of the absolute value. If $\left|y_{1}\right|<\left|y_{2}\right|, l_{1}=0.618 l, x_{3}=x_{2}-l_{1}, y_{3}=F\left(x_{3}\right)$. On the contrary, if $\left|y_{1}\right| \geq\left|y_{2}\right|$,then $l_{1}=0.618 \mathrm{l}, x_{3}=x_{1}+l_{1}, y_{3}=F\left(x_{3}\right)$. Second division, compare the symbols of $y_{1}, y_{3}$ and $y_{2}, y_{3}$, in the opposite position by segmentation, get the length of the smaller area of $l_{2}=0.618 l_{1}=0.618^{2} l$, the length of the $n$-th is $l_{n}, l_{n}=0.618 l_{n-1}=0.618^{n} l, n+1$ times segmentation was obtained length of $l_{n+1}$, then $l_{n+1}=0.618 l_{n}=q_{\varepsilon} 618^{n+1} l$, after segmentation of $\mathrm{n}$ times, $0<l_{n}=0.618^{n} l \leq 1 e-8, n=\log 0.618^{\frac{b-a}{b-a}}$. For all $n, n$ iterations can get points $x_{n}$, then $\lim _{n \rightarrow \infty} y=F\left(x_{n}\right)=0$. Interval iterative process is shown in Fig. 1.

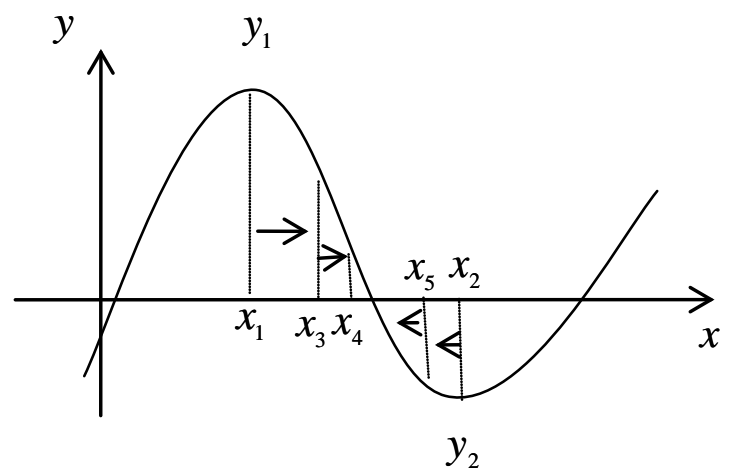

Fig. 1. Interval iterative process

\section{Numerical Results}

This section reports numerical results of large number experiments for solving formula (1). Our numerical experiments are running on a PC Intel Pentium IV of $2.93 \mathrm{GHz}$ CPU.

For $f_{n}(x)=\sum_{i=0}^{3 n} a_{n, i} x^{i} \quad$,then

$$
a_{n, i}= \begin{cases}\sum_{j=0}^{i} \beta_{n, 2-j} a_{n-1, j}-\sum_{j=0}^{i} \alpha_{n, 2-j} a_{n-2, j} & \text { if }(i<3) \\ \sum_{j=0}^{3} \beta_{n, 3-j} a_{n-1, i+j-3}-\sum_{j=0}^{2} \alpha_{n, 2-j} a_{n-2, i+j-2} & \text { elseif }(2<i<3 n-5) \\ \sum_{j=0}^{3} \beta_{n, 3-j} a_{n-1, i+j-3}-\sum_{j=0}^{1} \alpha_{n, 2-j} a_{n-2, i+j-2} & \text { elseif }(i=3 n-5) \\ \sum_{j=0}^{3} \beta_{n, 3-j} a_{n-1, i+j-3}-\sum_{j=0}^{0} \alpha_{n, 2-j} a_{n-2, i+j-2} & \text { elseif }(i=3 n-4) \\ \sum_{j=0}^{3 n-i} \beta_{n, 3-j} a_{n-1, i+j-3} & \text { elseif }(i>3 n-4)\end{cases}
$$




$$
\begin{aligned}
& \alpha_{n, i}=\left(48 i^{2}-96 i+16\right) n^{2+i} \\
& a_{0,0}=1, a_{0,1}=0 .
\end{aligned}, \quad \beta_{n, i}=\left\{\begin{array}{ll}
\left(\frac{28}{3} i^{2}-\frac{92}{3} i+8\right) n+\frac{10}{3} i^{2}-\frac{38}{3} i+4 & \text { if }(i \neq 1) \\
-4\left(2 n^{2}+2 n-1-\mathrm{A}\right) & \text { else }
\end{array}\right. \text {, the parameter for }
$$

\begin{tabular}{|c|c|c|c|}
\hline Parameter A & \multicolumn{3}{|c|}{ Real solutions } \\
\hline 2 & $\begin{array}{l}x 1=-82943.70783981 \\
285 \\
x 2=-137.4794816813 \\
8 \\
x 3=-24.62941244138 \\
x 4=0.25464464090\end{array}$ & $\begin{array}{l}x 5=5.84390994195 \\
x 6=6.73918581767 \\
x 7=11.96467086181 \\
x 8=13.16994387815 \\
x 9=15.89617977254 \\
x 10=29.33345593633\end{array}$ & $\begin{array}{l}x 11=30.19235325208 \\
x 12=31.05308363598 \\
x 13=31.91557758080 \\
x 14=32.77977148313 \\
x 15=33.64560627900\end{array}$ \\
\hline 5 & $\begin{array}{l}x 1=-46656.92469148 \\
775 \\
x 2=-166.3189677710 \\
7 \\
x 3=-24.01470653335 \\
x 4=0.25462393252\end{array}$ & $\begin{array}{l}\mathrm{x} 5=5.96854710435 \\
\mathrm{x} 6=6.19878567390 \\
\mathrm{x} 7=11.12981907392 \\
\mathrm{x} 8=12.21589799631 \\
\mathrm{x} 9=12.82985813600 \\
\mathrm{x} 10=15.47980264381\end{array}$ & $\begin{array}{l}\mathrm{x} 11=16.41357325085 \\
\mathrm{x} 12=28.21260927667 \\
\mathrm{x} 13=30.88489498745 \\
\mathrm{x} 14=31.77898160173 \\
\mathrm{x} 15=34.47015922101 \\
\mathrm{x} 16=35.36998452745\end{array}$ \\
\hline 13 & $\begin{array}{l}x 1=-6231.369117926 \\
04 \\
x 2=-346.4863752826 \\
0 \\
x 3=-23.26486707092 \\
x 4=0.25456913449 \\
x 5=6.45617082537\end{array}$ & $\begin{array}{l}x 6=11.49177431256 \\
x 7=20.74321461244 \\
x 8=44.76555219859 \\
x 9=-0.97164275378 \\
x 10=4.30228530599 \\
x 11=10.11562528432 \\
x 12=19.50641181729\end{array}$ & $\begin{array}{l}\mathrm{x} 13=43.66684844615 \\
\mathrm{x} 14=42.56857590795 \\
\mathrm{x} 15=41.47076278576 \\
\mathrm{x} 16=14.01345887746 \\
\mathrm{x} 17=39.27664031093 \\
\mathrm{x} 18=38.18040092904\end{array}$ \\
\hline-20 & $\begin{array}{l}\mathrm{x} 1=-1923192.308779 \\
24080 \\
\mathrm{x} 2=-46.32271103626 \\
\mathrm{x} 3=-36.49838473004 \\
\mathrm{x} 4=0.25479720617\end{array}$ & $\begin{array}{l}x 5=5.37015222972 \\
x 6=13.17869604286 \\
x 7=16.22028174510 \\
x 8=26.86050635490 \\
x 9=26.12389925758\end{array}$ & $\begin{array}{l}x 10=13.14007060069 \\
x 11=24.66098042281 \\
x 12=22.49667175223 \\
x 13=20.37244933996 \\
x 14=18.28011606463\end{array}$ \\
\hline-6 & $\begin{array}{l}x 1=-307401.5508710 \\
5284 \\
x 2=-90.63238180991 \\
x 3=-27.23270656400 \\
x 4=0.25470024428 \\
x 5=5.59747778945\end{array}$ & $\begin{array}{l}x 6=7.56693167456 \\
x 7=11.83812611401 \\
x 8=13.50820889113 \\
x 9=15.53239698026 \\
x 10=20.91491978177 \\
x 11=25.59246428463\end{array}$ & $\begin{array}{l}\mathrm{x} 12=27.17476234184 \\
\mathrm{x} 13=28.76753458244 \\
\mathrm{x} 14=29.56767978986 \\
\mathrm{x} 15=30.37023258871 \\
\mathrm{x} 16=26.38227103129\end{array}$ \\
\hline
\end{tabular}

Table.1. Numerical results

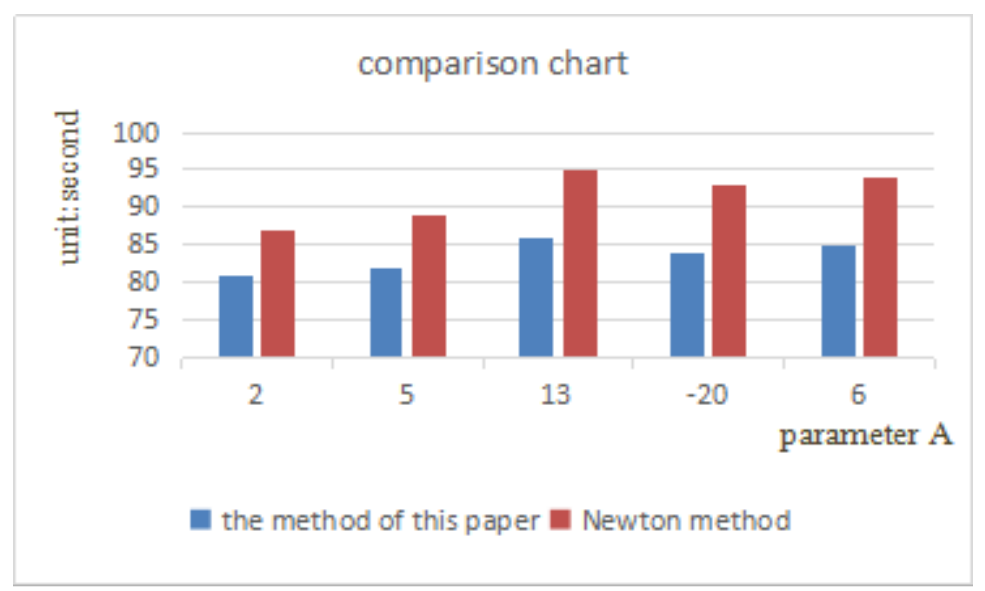

Fig. 2 Time contrast chart

Our numerical results are reported in table 1, which obtained all real solutions for parameter A. Fig. 2 is the time comparison chart that the method of this paper and Newton method. As can be seen 
from Fig.2, this method is superior to the Newton method in solving the efficiency, that is, the golden section method can effectively reduce the number of iterations. All real solutions are time consuming and short, which indicates that the parallel computation can be used to improve the efficiency of solving higher order equations. This method has global convergence, and the convergence speed is fast, solving process can meet the requirements of high efficiency and fast, and get real solution precision is higher.

\section{Conclusion}

Parallel computation is an advantage to solve the complicated computation problem, and the golden section method can effectively reduce the number of iterations. The combination, give full play to their advantages, makes the solving process has strong global convergence and searching efficiency of all real solutions for improved significantly. Numerical experiment results show that the method not only in theory, at the same time is effective in the actual calculation. And because it does not depend on the choice of initial values, can ensure that all real number obtained equations, can be widely used in practical engineering computing.

\section{References}

[1] M.Yu.Kokurin,A.I.Kozlov,"On a posteriori approxinmation of the set of solutions to a system of quadratic equations using Newton’s method ,"Numerical Analysis and Applications,vol.7,no.1, pp.45-56,2014.

[2] B.S.Goh,D.B.McDonald,"Newton Methods to Solve a System of Nonlinear Algebraic Equations,"Journal of Optimization Theory and Applications, vol.164, no.1, pp.261-276, 2015.

[3] Lixia Liu,Sanyang Liu,Yan Wu, "A smoothing Newton method for symmetric cone complementarity problem," Journal of Applied Mathematics and Computing, vol. 47, no. 1, pp. 175-191, 2015.

[4] X.B.Xu,Y.H.Ling,"Senilocal convergence for a family of Chebyshev-Halley like iterations under a mild differentiability condition", vol. 40, no. 1, pp. 627-647, 2012.

[5] X.H.Wang,J.S.Kou,C.Q.Gu, "Semilocal Convergence of a Class of Modified Super-Halley Methods in Banach Spaces," Journal of Optimization Theory and Applications, vol. 153, no. 3, pp. 779-793, 2012.

[6] Ramandeep Behl,V.Kanwar,KapilK.Sharma."Optimal equi-scaled families of Jarratt's," International Journal of Computer Mathematics, vol. 90,no. 2,pp. 408-422, 2013.

[7] Peng Cai,Tingmei Wang,Qihua Wang."Formulation Optimization of Friction Material with Golden Section Approach," Tribology Transactions, vol. 59, no. 1, pp. 28-32, 2016.

[8] K.C.Huang,Y.L.Tsai,Hsiao-Ching liu, "Task ranking and allocation in list-based workflow scheduling on parallel computing platform," The Journal of Supercomputing, vol. 71, no. 1, pp. 217-240, 2015.

[9] J.H.Kang,S.J.Kang,"Line recognition algorithm for 3D polygonal model using a parallel computing platform,"Multimedia Tools and Applications, vol.74, no.1, pp.259-270, 2015.

[10] Z.Y.Mo,A.Q.Zhang,Y.Zhang,"A new parallel algorithm for vertex priorities of data flow acyclic digraphs,"The Journal of Supercomputing, vol. 68, no. 1, pp.49-64, 2014.

[11] J.Chung,H.J.Jang,K.H.Jung,etc, "Parallel approach for processing itinerary-based RNN queries in object track WSNs," Telecommunication System, vol. 55, no. 1, pp. 55-69, 2014.

[12] Y.L.Liu,P.R.Fu, "Lectures on mathematical analysis,"Beijing:Higer Education Press,1997. 